\title{
Deplete Abide Time And Inflammation. Growth, During Fringe Saphenous Impoverish Managing
}

\author{
Dr. Rohit Yadav, Dr. Shrawan Patel
}

Pandit Bhagwat Dayal Sharma Institute Of Medical Sciences India

\begin{abstract}
Fringe Depletes have been crucial instruments of present day medication. Albeit fringe applications can be utilized for a huge number of purposes, these applications may cause intricacies, some of which have genuine impacts. Of these inconveniences, the most regularly watched is phlebitis. This investigation was directed to decide the impact of Deplete inhabit time on inflammation improvement during fringe Deplete organization.
\end{abstract}

Techniques: This investigation decided the impact of Deplete inhabit time on inflammation advancement during fringe Deplete organization. The investigation incorporated a sum of 103 people who were controlled 439 Depletes and fulfilled the examination enlistment criteria at one irresistible sicknesses center in Istanbul/India. Information were incorporated from Patient Information Forms, fringe Deplete and Therapy Information Forms, revealed evaluations dependent on the Visual Infusion inflammation Assessment Scale, and fringe Deplete Nurse Observation Forms. The information were broke down utilizing SPSS. Results: The mean patient age was $53.75 \pm 15.54$ (standard deviation) years, and $59.2 \%$ of the investigation members were men.inflammationwas distinguished in $41.2 \%$ of fringe fringe Depletes, and the rate diminished with expanded Deplete inhabit time. Investigations demonstrated that Deplete inhabit time, antiinfection use, sex, and Depleteization destinations were essentially connected with improvement of phlebitis. End: The aftereffects of this investigation demonstrate that Depletes can be utilized for longer timeframes when directed under ideal conditions and with fitting reconnaissance.

Keywords: fringe venous Deplete, Deplete inhabit time, Phlebitis, Thrombophlebitis 


\section{Introduction}

Inpatients are presented to numerous mediations for finding and treatment. Organization of parenteral drug is the most usually utilized mediation technique. Over $80 \%$ of inpatients get fringe (IV) treatment by addition of a Deplete into the vein.1-3managing of parenteral medication is an essential piece of nursing. Attendants direct fringe fluids or meds endorsed by specialists or clinicians to patients. Attendants additionally screen and care for patients.4,5 Fringe Depletes are essential apparatuses of present day drug. Depletes are vital for organization of fluid medications and blood and blood items, just as for parenteral nourishing, close checking, and mixture of different drugs. In spite of the fact that IVs have a large number of employments, they can cause intricacies that may prompt significant issues, for example, extravasation, ecchymosis, hematoma, disease, and phlebitis. The most widely recognized among these complexities is phlebitis.1-3,6-9 Phlebitis, defined as inflammation of the venous tunica intima, is a typical yet avoidable entanglement in people accepting IV medicines. It is by and large connected with fringe venous Deplete (PVC) use.inflammationis brought about by different factors, for example, wide, thick, and long Deplete lumens; Deplete material organization; inclusion site; number of additions; inhabiting length; concentration of medicine

and solutions; flow rate; contravention of aseptic strategies; kind of covering utilized for adjustment; and the pace of implantation set trade.

\section{Strategies}

Study point and sort of research: A cross-sectional investigation was led between May 2011November 2011 to decide the impact of Deplete inhabit time on the advancement of inflammation during PVC. Members: A sum of 439 Depletes were regulated to 103 patients who coordinated the examination criteria, including grown-ups more seasoned than 18 years old willing to collaborate, convey, and take an interest in the investigation and who got IV treatment during hospitalization. Patients in the irresistible ailments center who were hospitalized for short of what one day, for example, the individuals who experienced liver biopsies, were barred from the investigation. Patients accepting chemotherapy were likewise prohibited. Information accumulation apparatuses: Data were gathered utilizing number of structures and evaluation frameworks, including a Patient Information Form, Information Form on fringe Venous Deplete and Treatment, Visual Infusion inflammation Assessment Scale (VIPAS) Staging Key for fringes, and the fringe Venous Deplete Nurse Observation Form, as portrayed underneath. The Patient Information Form included statistic highlights, for example, age, sex, and restorative conclusion. The creators built up the Information Form on fringe Venous Deplete and Treatment after audit of pertinent writing. The structure gathered information on the quantity of Depletes; anatomical 


\section{PHARMACEUTICAL RESEARCH}

site and recurrence of Deplete organization per site; use of antibiotics and fluids; duration of Deplete stay in the vein; inflammation advancement ;inflammation level; and whether the Deplete has instruments like triple taps, vein valve, and Dose-stream

VIPAS group's inflammation into 5 evaluations: Grade 1 has none of the run of the mill side effects of phlebitis, for example, torment, rash, and edema. Just Deplete perception is suggested. Evaluation 2 depicts the early side effects of phlebitis, for example, torment upon palpation and a rash under $2.5 \mathrm{~cm}$ in width around the Deplete site. VIPAS proposes Deplete substitution. Evaluation 3inflammationalludes to mid-level sickness: IV site rashes are somewhere in the range of 2.5 and $5 \mathrm{~cm}$, and palpation of the IV site uncovers agony and inflexibility. Treatment ought to be considered after Deplete substitution and specialist notification. Grade 4

phlebitis is advanced phlebitis or the beginning of thrombophlebitis. At this level, there is a rash bigger than $5 \mathrm{~cm}$ around the IV site and torment and unbending nature upon palpation. VIPAS suggests Deplete substitution, specialist counsel, and thought of treatment. At long last, grade 5inflammationdepicts propelled thrombophlebitis. This evaluation incorporates grade 4 side effects just as purulent waste. Proposals incorporate treatment thought after Deplete substitution and specialist.

\section{Results}

Patients directed anti-infection agents through Depletes had 2.4 occasions the danger of creating phlebitis. Patients who got absolute and other parenteral nourishment (hepatamine, 10\% dextrose) as an IV fluid developed phlebitis 3 times more often than patients who were managed $5 \%$ dextrose, isolate, $0.9 \% \mathrm{NaCl}$, blood and blood items, or no liquid.

The Effect of Seven Variables on inflammation Development because of PVC managing: Analysis of the impacts of 7 factors related with inflammation demonstrated that Deplete inhabit time has the best impact $(p=0.000)$, trailed by utilization of anti-microbial $(p=0.002)$, understanding sex $(p=0.007)$, and Depleteization site $(p=0.034)$. These factors are recorded concurring to the Wald test results for the statistical significance of the regression coefficients 
Discourse fringe Depletes, utilized for both treatment and care, make it conceivable to oversee liquid electrolytes, blood and blood products, medicine, and parenteral nourishment, and to access veins for hemodynamic perception. Phlebitis, the most widely recognized confusion, influences $75 \%$ of inpatients. Phlebitis is a significant

clinical problem. It contrarily influences the solace of the patient, the length of Deplete use, the hospitalization time frame, and treatment costs. This expressive cross-sectional investigation broke down an aggregate of 103 patients managed 439 Depletes to decide the impact of PVC length on the improvement of phlebitis. Factors, for example, age, sex, number of Depleteizations, Deplete connection destinations, recurrence of mediations, and organization of antibiotics and parenteral fluids through Depletes can influence the improvement of phlebitis. Along these lines, these factors are incorporated into this examination.

PVCs developed phlebitis in $41.2 \%$ of subjects. Significant differences in phlebitis stage distribution were saw between gatherings. Propelled examinations demonstrated the most astounding frequency rate in Depletes embedded for 48 hours or less, with a diminished rate in Depletes embedded for 4996 hours, and the least rate in Depletes staying set up for 97-120 hours. In a booklet distributed in 2011, the Centers for Disease Control and Prevention pointed out 2 identified with PVC span

\section{References}

1. Aygün G, Kardashian K, DikmenY, YaşarH, Midilli K, Can G, et al. Assessment of fringe venous Deplete contamination in emergency unit. J Infect Dis Clin Microbial..

2. Slushy E. Assessment of inflammation improvement and effecting factors during fringe Deplete situation. Unpublished Master's Thesis, Dukes Ell University, Institute of Health Sciences 2002;517.

3. Dakar V. Disease control rehearses at fringe venous Depletes and intravascular Depletes. J Knurs Educe Res.

4. Celia Z, Anil C. Confusions of fringe organization. Day by day J Gastroenterology..

5. Abbasoğlu Access Routes in Support Treatment of Parenteral Nutrition. India Pandit Bhagwat Dayal Sharma Institute Med Sci. 2007; 3(18):32-44. 


\section{THE AMERICAN JOURNAL OF}

MEDICAL SCIENCES AND

VOLUME01 ISSUE02

\section{PHARMACEUTICAL RESEARCH}

6. Roper N, Logan WW, Tierney AJ. A Model for Nursing Based On a Model of Living. Fourth version. New York: Churchill Living Stone Publish; 2000. 